\title{
The use of ultrasonography in learning clinical examination-a pilot study involving third year medical students
}

\section{Daniela Fodor ${ }^{1}$, Radu Badea ${ }^{2}$, Laura Poanta ${ }^{1}$, Dan Lucian Dumitraşcu ${ }^{1}$, Anca Dana Buzoianu ${ }^{3}$, Petru-Adrian Mircea ${ }^{4}$}

${ }^{1} 2^{\text {nd }}$ Internal Medicine Department, ${ }^{2}$ Department of Ultrasound, Regional Institute of Gastroenterology and Hepatology ${ }^{3}$ Department of Clinical Pharmacology, ${ }^{4} 1$ st Internal Medicine Department, "Iuliu Hatieganu" University of Medicine and Pharmacy, Cluj-Napoca, Romania

\begin{abstract}
Background: Physical examination is a crucial part in medical student curricula, but a decline in physical examination skills was noted in the last two decades. The benefic role of ultrasonography (US) as an adjuvant method for clinical examination was evaluated in many studies, but there are different approaches among countries and universities. The aim of this study was to evaluate the role of US in learning physical exam in $3^{\text {rd }}$ year medical students. Methods: One hundred and four 3rd year medical students were randomly enrolled. They were divided first in two equal groups (I and II, with and without US, by rotation) and then in smaller groups of 8-9 students. Pairs between smaller groups belonging to group I and II were randomly established in order to be trained and evaluated by the same instructor. We verified the influence of US on the correctness of thyroid palpation, lung percussion (inferior limit of the lung), and liver size estimation. They received no special training on US. After the learning sessions (four sessions, one hour each) a questionnaire was applied to each student. Results: For thyroid palpation, there were no significant differences regarding the gland dimensions. For lung and liver palpation the results showed better results for some points in groups using US as adjuvant, but the most significant difference regarded the appreciation of self confidence (in favor of US-groups). The majority of the students using US appreciated as very useful the informations provided by US. Also, we found a clear improvement in detecting the upper limit of the liver comparing with the inferior limit of the lung, as result of better clinical skills. Discussions and conclusion: The results from this pilot study proved the utility of US in learning clinical examination. The students can better understand the regional anatomy and can confirm immediately the physical findings. It is important for students to reach a certain level of knowledge regarding physical exam to better understand the role of US. Overall, we can state that use of US in the daily clinical examination teaching greatly improved the students' clinical examination abilities and that is a strong argument for integrating basic US into the medical school curriculum.
\end{abstract}

Keywords: clinical examination, ultrasonography, medical student education

The traditional methods for clinical examination are inspection, palpation, percussion, and auscultation, teaching physical examination (bedside examination of the patient) being an important part in medical schools students curriculum. The ability to perform a competent physical examination is considered to be an essen-

Received 10.06.2012 Accepted 24.06.2012

Med Ultrason

2012, Vol. 14, No 3, 177-181

Corresponding author: Petru-Adrian Mircea

41st Internal Medicine Department

3-5 Clinicilor str

Cluj-Napoca 400006, Romania

Phone: 0040741553391, Fax: 0040264590899

Email: petrumircea@yahoo.com tial clinical skill for medical students and this must be achieved especially during the practical clerkships. Over the last two decades, a decline in physical examination skills of medical students, interns, and residents was noted [1-6]. The fact was attributed to the greater availability of advanced technologies [7], increased reliance on high technology diagnostic tests, or lack of supervised bedside instruction $[6,8]$.

In this setting, new modalities for help learners in acquire competence and confidence in physical examination skills, were searched. Ultrasonography (US) is a non-invasive imagistic technique, which has several advantages over other imaging techniques, including low cost, easy and rapid performance. The usefulness of US as diagnostic tool is generally and largely recognized. 
The role of US as an adjuvant method to the clinical examination or anatomy learning in medical schools was evaluated in different studies [9-15], demonstrating improvement in physical examination skills or in anatomic knowledge of the students. The terms as "living anatomy" or "visual feedback of clinical examination" were used referring to the US $[10,12]$.

Medical education curriculum has differences between countries and universities. In Romanian Medical Schools clinical examination skills are learned during the third year of study (in Romania the medical school last for 6 years) during Medical Semiology and Surgical Semiology programs. Taking into account the advances in US technology (portable machines, easy manageable) and the experiences of other medical schools $[13,16]$ we initiated a pilot study involving third year medical students. Our aims were 1) to determine if the use of US (visual medicine), as adjuvant of clinical examination, influence the correctness of different clinical techniques (thyroid palpation, lung percussion, and liver examination); 2) to evaluate if the use of US during the normal school schedule has any influences on the learning quality; 3 ) to appreciate the students' opinions on the utility of the program.

\section{Material and method}

The study was approved by the Ethical Committee of the "Iuliu Hatieganu" University of Medicine and Pharmacy, Cluj-Napoca, Romania and by the Dean of Faculty of Medicine. Written, informed consent for participation was obtained from each student prior to enrolment. Also, written, informed consent was obtained from patients examined during the educational program.

One hundred and four $3^{\text {rd }}$ year medical students were randomly enrolled (approximately $20 \%$ of the total number of $3^{\text {rd }}$ year students). The students were divided first in 2 groups of 52 students (group I and II) and then in smaller groups of 8-9 students. Pairs between smaller groups belonging to group I and II were randomly established in order to be trained and evaluated by the same instructor from our staff (one teacher had to instruct one smaller group from group I and one smaller group from group II). This distribution was maintained during the whole study. All the staff members involved into this project had at least medium knowledge about US.

During the school year all the students follow the same program as the whole $3^{\text {rd }}$ year students, no intervention into the educational program was made.

We establish to verify the influence of US on the correctness of clinical examination using 3 items: thyroid palpation, lung percussion (inferior limit of the lung), and liver size estimation. Also, these verifications were made at the moment when these techniques are in schedule to be learned (the first two in the first semester and the last in the second semester). All students received the traditional instruction in palpation and percussion of the thyroid, lung, and liver, in normal and pathological cases (reading, staff practical demonstration, and self practice).

Considering that is unacceptable to deny students exposure to a potential beneficial educational intervention [10] we decided not to use a placebo group control. For this reason, students from group I learned thyroid palpation and liver examination using only clinical skills and students from group II verified the clinical examination by using US. For lung percussion group I used US to verify the clinical finding and group II used only clinical skills.

Students received no special training in US. At the bedside, simple explanation about the US imagines were offered ("black is fluid, white is air or bone, and gray is a parenchymatous structure- thyroid or liver") and they were learned to identify the movements of the lung and liver during inspiration/expiration or of the thyroid during swallowing (real-time examination).

Ultrasoud equipment consisted of portable GE Venue 40 US devices equipped with $12 \mathrm{MHz}$ linear and 3.5 $\mathrm{MHz}$ convex transducers.

For learning clinical examination (with or without US as adjuvant) four training sessions, one hour long each, were dedicated for every item. At the end the students were evaluated on the same patient, in the same day, and a dedicated questionnaire was fullfiled. Comparisons were made between the smaller groups instructed by the same teacher and finally between the two groups.

For thryoid cuantification, the longitudinal diameters of the lobes (distances between clavicles and upper point of the palpable lobes) and transverse diameter (distance between palpable edges at thyroid isthmus level) were measured using a ruler. The results were compared with the standard limits determined by US by a staff member.

For inferior limit of the lung assessment the percussion of the right anterior thorax along the 3 lines was performed: parasternal, mid-clavicular, and anterior axillary. The limit between lung resonance and liver dullness was noted (P1, P2, P3) and the distance between this limit and an arbitrary point prior established was measured. The results were compared with gold standard limits determined by US.

Liver size estimation was made along the right midclavicular line, establishing by percussion the superior limit and by a combination of palpation-percussion the inferior limit. Liver size was considered to be the dis- 
tance between the two points. The results were compared with standard limits determined by US by a staff member.

Statistical analysis was performed using $Z$ test for comparing proportions from two independent groups ("two sample" test) at significance level $\alpha=5 \%$ and confidence interval $95 \%$.

\section{Results}

First item- thyroid palpation (group I using only clinical skills and group II using US as adjuvant of clinical examination)

No significant differences between groups $(\mathrm{p}>0.05)$ were obtained when compared the clinical findings at inspection and palpation, thyroid measurements, and appreciation of self difficulty in thyroid palpation. Concerning the confidence in clinical skills, group I had modest confidence in self palpation skills vs. high confidence of group II $(\mathrm{p}<0.001)$. Group I appreciated the time allocated for thyroid palpation learning as being insufficient/ indifferent comparing with group II which appreciated the time as being totally sufficient $(\mathrm{p}<0.05)$.

In group II, $82 \%$ of the students appreciate as useful/necessary the supplementary information given by US and $96 \%$ of students considered that US improved their thyroid palpation skills (64\% of them appreciated as highly improvement).
Second item- inferior limit of the lung (group I using US as adjuvant of clinical examination and group II using only clinical skills)

The number and proportion of students that correctly identified the inferior limit of the lung along the parasternal, mid-clavicular, and anterior axillary lines are mentioned in table I.

None of the students correctly identified all 3 points. From 156 measurement to be done by every group (52 students, each 3 points), 41 (26.3\%) of measurement in group II and $22(14.1 \%)$ in group I had correct values $(\mathrm{p}=0.0067)$.

No significant differences across groups $(\mathrm{p}>0.05)$ was obtained when difficulty of procedures, confidence in skills, and time assingned for instruction was evaluated.

In group I, 69\% of the students appreciate as useful/ necessary the supplementary information given by US and $60 \%$ of students considered that US improved their performance in lung percussion.

Third item- liver size measurement (group I using only clinical skills and group II using US as adjuvant of clinical examination).

No significant difference was obtained between groups in liver superior limit identification, but the inferior limit of the liver was correctly identified significantly more often in group II (table II).

Table I. The correct identification of the inferior limit of the lung

\begin{tabular}{lccc}
\hline $\begin{array}{l}\text { Inferior limit } \\
\text { of the lung }\end{array}$ & $\begin{array}{c}\text { Group I (US as adjuvant) } \\
\text { n (\%) }\end{array}$ & $\begin{array}{c}\text { Group II (clinical skils) } \\
\text { n (\%) }\end{array}$ & $\boldsymbol{P}$ \\
\hline P1 & $15(29)$ & $13(25)$ & 0.6578 \\
P2 & $14(27)$ & $7(13)$ & 0.0829 \\
P3 & $12(23)$ & $2(4)$ & $\mathbf{0 . 0 0 2 8}$ \\
P1+P2 & $5(10)$ & $4(8)$ & 0.7262 \\
P1+P3 & $7(13)$ & $1(2)$ & $\mathbf{0 . 0 2 3 7}$ \\
P2+P3 & $4(8)$ & $0(0)$ & $\mathbf{0 . 0 3 7 4}$ \\
P1+P2+P3 & $0(0)$ & $0(0)$ & 0.9999 \\
\hline
\end{tabular}

n- number of students that correctly identified the points

Table II. Correct identification of the superior and inferior limit of liver

\begin{tabular}{lccc}
\hline Liver & $\begin{array}{c}\text { Group I (clinical skils) } \\
\text { n (\%) }\end{array}$ & $\begin{array}{c}\text { Group II (US as adjuvant) } \\
\text { n (\%) }\end{array}$ & P \\
\hline Superior limit & $23(44 \%)$ & $27(52 \%)$ & 0.4127 \\
Inferior limit & $7(13 \%)$ & $25(48 \%)$ & $<\mathbf{0 . 0 0 0 1}$ \\
\hline
\end{tabular}

n- number of students that correctly identified the points 
Nineteen (37\%) students from group II and 4 (8\%) from group I correctly identified both limits of the liver $(\mathrm{p}<0.00001)$. The inferior limit of the liver was overestimated more frequently in group I (28 students-53.8\%) and underestimated more frequently in group II (21 students-40.3\%).

No significant differences between groups $(p>0.05)$ was obtained when appreciation of the degree of difficulty of procedures was evaluated. Self reported confidence was high in $11.54 \%$ students from group II vs. $0 \%$ from group I ( $p<0.05$ ), and time assingned for instruction was considered insufficient by $38.46 \%$ students from group I $(\mathrm{p}<0.05)$.

In group II, $81 \%$ of students appreciated as useful/ necessary the supplementary information given by US and $81 \%$ of the students considered that the use of US highly improved their clinical skills.

\section{Discussions}

The idea of introducing US in medical school curriculum (anatomy study, clinical examination of the abdomen or heart, or emergency medicine) is not new. Tshibwabwa et al [17] and Wicke et al [18] used US during anatomy sessions to enhance the students' knowledge of anatomy and the open feedback reported indicated that students wanted to attend more courses of this sort. Teichgraber et al [15] reported an improvement in anatomical knowledge in $48.8 \%$ of the students involved in seminar based on US imagines. Shapiro et al [14] evaluated 5 medical students while performing both ultrasound and physical examination on patient. Results of a student survey showed a favorable response to using US as an aid to teaching physical examination. Butter et al [10] study concluded that US may boost the students' level of skill in abdominal examination once they achieved the basic technique. In Rao et al [13] pilot study, $83 \%$ of students agreed or strongly agreed that their experience with US education was positive.

The importance of US in educational program is underline by incorporation of US in medical student curricula in some University of Medicine (Wayne University or South Carolina University in the USA) because they concluded that the ultrasound experience enhances the students' medical education $[13,16]$.

The results from this pilot study proved the utility of US in learning clinical examination during the $3^{\text {rd }}$ year of medical school. US technology has two major didactical positive advantages: familiarity of the modern students in using knobs, buttons or controller, which make more natural the use of a device such a US machine and the quality of US to be used as visual medicine. US offers students the ability to "visualize" the physical findings of their clinical examination. The student can better understand the regional anatomy and can confirm immediately the physical findings, US being from this point of view a perfect tool to aid students in their learning.

The thyroid palpation was the first item evaluated. No significant differences were obtained at thyroid measurement and we explained that to the fact that the study raised the competitive atmosphere between groups that leaded to an increased attention and concern for the correct clinical examination. In fact, this competitive atmosphere lasted for the whole study period, the staff teachers underlining the increased interest of students in learning clinical examination. Also, the teachers found easier to conduct the educational program as comparing with anterior generations. The higher confidence in their own clinical examination found in US group is an important tool for better results in their future professional life.

In lung percussion, better results were found in US group. Interesting conclusion comes when compare the P2 from lung percussion (second item, group I using US) and the superior limit of the liver (third item, group II using US), in both cases using the same technique for examination. In the first case only $20 \%$ of students correctly identified $\mathrm{P} 2$ while in the second, $48 \%$ of students correctly found it. This fact could be interpreted as the need for students to reach a minimal level of knowledge and practical skills, before to apply correctly the information provided by US. The need for a minimal level of competence before any benefit from the visual feedback offered by US was also the conclusion of Butter et al [10] for abdominal examination. Interesting, in our study the underestimation of the liver size was more frequently observed in the group that used US. The same report is found in Butter et al [10] study and they explained this discrepancy as the result of poor technique and inexperience. In our study this results could be explained also by the fact that students were instructed to see in real time (by US) the movement of the liver during inspiration/ expiration. Probably these movements were exaggerated represented in their mind. We can assume that a longer instruction time for liver examination would have been more beneficial in training and results.

We were very pleased to see that the great majority of the students found useful/necessary the information offered by US and the use of US highly improved their clinical skills. In this setting we think that US is a perfect tool to aid students in their learning.

Our study has several limits. First of all, lack of any anterior experience/knowledge in US of our students and the limited time for teaching. During the study, we imposed no rule about self-information about US or clini- 
cal examination. Second, the inherent differences existed between teachers could influenced the students' results. Finally, we did not determinate the students' ability in detecting pathology, the final goal for the future doctors.

In conclusion, our pilot study showed that the students using US as an adjuvant method in learning clinical examination yielded significantly superior results than their counterparts who only used the traditional methods for physical examination. We can therefore say that use of US in the daily clinical examination teaching greatly improved the students' clinical examination abilities and that is strong argument for integrating basic US into the medical education curriculum.

\section{Conflict of interest: none}

Acknowledges: GE for providing the technological infrastructure for the project; Dr. Sorana Bolboaca for the statistical evaluation of the data; staff teachers: Simona Grad, Cosmin Grad, Mihai Porojan, Flaviu Rusu, and Aura Sinpetrean.

\section{References}

1. Mangione S, Nieman LZ. Cardiac auscultatory skills of internal medicine and family practice trainees. A comparison of diagnostic proficiency. JAMA 1997; 278: 717-722.

2. Mangione S, Nieman LZ. Pulmonary auscultatory skills during training in internal medicine and family practice. Am J Respir Crit Care Med 1999; 159: 1119-1124.

3. Marcus GM, Vessey J, Jordan MV, et al. Relationship between accurate auscultation of a clinically useful third heart sound and level of experience. Arch Intern Med 2006; 166: 617-622.

4. Sachdeva AK, Loiacono LA, Amiel GE, Blair PG, Friedman M, Roslyn JJ.Variability in the clinical skills of residents entering training programs in surgery. Surgery 1995; 118: 300-308.

5. Vukanovic-Criley JM, Criley S, Warde CM, et al. Competency in cardiac examination skills in medical students, trainees, physicians, and faculty: a multicenter study. Arch Intern Med 2006; 166: 610-616.
6. York NL, Niehaus AH, Markwell SJ, Folse JR. Evaluation of students' physical examination skills during their surgery clerkship. Am J Surg 1999; 177: 240-243.

7. Simel DL.Time, now, to recover the fun in the physical examination rather than abandon it. Arch Intern Med 2006; 166: 603-604.

8. Alexander EK. Perspective: moving students beyond an organ-based approach when teaching medical interviewing and physical examination skills. Acad Med 2008; 83:906909.

9. Barloon TJ, Brown BP, Abu-Yousef MM, et al. Teaching physical examination of the adult liver with use of real-time sonography. Acad Radiol 1998; 5: 101-103.

10. Butter J, Grant TH, Egan M, et al. Does ultrasound training boost Year 1 medical student competence and confidence when learning abdominal examination? Med Educ 2007; 41: 843-848.

11. Fernández-Frackelton M, Peterson M, Lewis RJ, Pérez JE, Coates WC. A bedside ultrasound curriculum for medical students: prospective evaluation of skill acquisition. Teach Learn Med 2007; 19: 14-19.

12. Ivanusic J, Cowie B, Barrington M. Undergraduate student perceptions of the use of ultrasonography in the study of "living anatomy". Anat Sci Educ 2010; 3: 318-322.

13. Rao S, van Holsbeeck L, Musial JL. A pilot study of comprehensive ultrasound education at the Wayne State University School of Medicine: a pioneer year review. J Ultrasound Med 2008; 27: 745-749.

14. Shapiro RS, Ko PK, Jacobson S. A pilot project to study the use of ultrasonography for teaching physical examination to medical students. Comput Biol Med 2002; 32: 403409.

15. Teichgräber UK, Meyer JM, Poulson Nautrup C, von Rautenfeld DB. Ultrasound anatomy: a practical teaching system in human gross anatomy. Med Educ 1996; 30: 296298.

16. Hoppmann RA, Rao VV, Poston MB, et al. An integrated ultrasound curriculum (iUSC) for medical students: 4-year experience. Crit Ultrasound J 2011; 3: 1-12.

17. Tshibwabwa ET, Groves HM. Integration of ultrasound in the education programme in anatomy. Med Educ 2005; 39 : 1148.

18. Wicke W, Brugger PC, Firbas W. Teaching ultrasound of the abdomen and the pelvic organs in the medicine curriculum in Vienna. Med Educ 2003; 37: 476. 